\title{
Theory of attosecond absorption spectroscopy in krypton
}

\author{
J. C. Baggesen, ${ }^{1}$ E. Lindroth, ${ }^{2}$ and L. B. Madsen ${ }^{1}$ \\ ${ }^{1}$ Lundbeck Foundation Theoretical Center for Quantum System Research, Department of Physics and Astronomy, \\ Aarhus University, DK-8000 Aarhus C, Denmark \\ ${ }^{2}$ Department of Physics, Stockholm University, AlbaNova University Center, SE-106 91 Stockholm, Sweden
}

(Received 25 November 2011; published 25 January 2012)

\begin{abstract}
A theory for time-domain attosecond pump-attosecond probe photoabsorption spectroscopy is formulated and related to the atomic response. The theory is illustrated through a study of attosecond absorption spectroscopy in krypton. The atomic parameters entering the formulation such as energies and Auger widths, as well as wave functions and dipole coupling matrix elements, are determined by accurate many-body structure calculations. We create a hole in a valence shell by an attosecond pump, couple an inner-shell electron to the hole by an attosecond probe, and then monitor the formation of the hole in this manner. In a second example, a hole is created in an inner shell by the first pulse, and the second probe pulse couples an even more tightly bound state to that hole. The hole decays in this example by Auger electron emission, and the absorption spectroscopy follows the decay of the hole and the associated coherences in real time.
\end{abstract}

DOI: 10.1103/PhysRevA.85.013415

\section{INTRODUCTION}

The advent of attosecond light pulses has opened the possibility of studying electron dynamics on its natural time scale. During recent years techniques have been developed that combine an XUV attosecond pulse and the intense laser field used for its production, to time-domain atomic and molecular processes. The latter laser field has typically a duration of a few femtoseconds and the frequency is in the IR domain. The needed synchronization of the XUV and IR pulses is inherent to the generation process and several schemes exist [1-6]. The use of the IR pulse to probe the dynamics initiated by the attosecond pulse is an adaptation to the low intensity of currently available pulses in the attosecond regime. The IR field is on the other hand truly strong and significantly perturbs the system under investigation. Although strong-field physics is interesting in its own right, less intrusive pulses would be an advantage for the investigation of attosecond dynamics. A desirable scenario would be if both pumping and probing could be made with XUV attosecond pulses. Not only would this be advantageous for the time resolution, the scaling of the ponderomotive energy with the inverse of the square of the light frequency would in addition mean that the atom is only weakly affected by the light field, even for the higher intensities hopefully available in the future. Here we investigate such a scenario through a numerical simulation. Transient absorption spectroscopy has been used for many years for longer pulses; see, e.g., [7-9]. To date, however, to our knowledge no transition absorption experiments have been performed with attosecond pulses. The signal relies on the absorption of two photons by the same atom: one from the pump beam and one from the probe. Both beams are relatively weak, so the signal is expected be low. Nevertheless, attosecond absorption spectroscopy seems the most promising avenue for time-resolving dynamics on the attosecond time scale by two attosecond pulses, since, by increasing the target density, this method may circumvent the problem posed by the weak pump and probe attosecond fields. We note that very recently intensities were reaches that allowed for the study of two-electron ejection by two-XUV-photon absorption using pulses with a duration around $1 \mathrm{fs}$ [10].
PACS number(s): $32.80 . \mathrm{Aa}, 78.47 . \mathrm{jb}, 82.53 . \mathrm{Kp}$

Our model example is core hole creation. The dynamics following the creation of a core hole includes rearrangement and decay processes, e.g., relaxation and emission of electrons (Auger effect) and photons. Apart from the obvious interest in these processes from a fundamental point of view they are of great interest for the study of radiation damage. The decay of a hole state was indeed one of the first targets for attosecond physics, when the Auger decay of a core hole in krypton was followed in the time domain [11]. The question of $\mathrm{x}$-ray absorption, leading to hole creation in a strong IR field, has also been addressed theoretically [12], and recently hole formation through x-ray pulses, as provided by contemporary and future free-electron laser facilities, was considered [13]. We consider here a different scenario. We imagine two attosecond pulses both weak enough that the light-matter interaction can be treated by perturbation theory. The first pulse is used to ionize the atom, and then the second pulse monitors by transient absorption spectroscopy whether or not a hole has been created. The wavelength of the second pulse is tuned to a transition from a deeper-lying core electron to the just emptied vacancy. By varying the delay between the two pulses, we will see that it is possible to study the formation of the hole by attosecond absorption spectroscopy.

The paper is organized as follows. In Sec. II we discuss the time-domain theory for absorption spectroscopy; then in Sec. III the response of the atom to the light pulses is detailed, while in Sec. IV we describe the calculation of the atomic parameters necessary to model this response. For this calculation we use relativistic many-body theory, leading to a realistic description of the atom and the singly charged ions created during the process. The results are discussed in Secs. V and VI concludes.

Atomic units $\left(\hbar=e=m_{e}=a_{0}=1\right)$ are used throughout the paper unless indicated otherwise.

\section{TIME-DOMAIN THEORY FOR ABSORPTION SPECTROSCOPY}

In absorption spectroscopy, one shines a laser pulse onto a target and measures the back action of the target on the 
electromagnetic field by monitoring the spectrum of the field after the interaction with the target. In principle, calculating this back action requires a full quantum-mechanical treatment of the target as well as of the electromagnetic field. Since this is a formidable task, we settle for a semiclassical model, where we solve the classical Maxwell wave equation approximately for the electromagnetic field while treating the target quantum mechanically. This procedure allows us to obtain an expression for the time- and position-dependent electromagnetic field which is related to the measured absorption spectrum in a simple manner [see Eq. (5) below].

The Maxwell wave equation for the electric field $\overrightarrow{\mathcal{E}}$ reads

$$
\left(\nabla^{2}-\frac{1}{c^{2}} \frac{\partial^{2}}{\partial t^{2}}\right) \overrightarrow{\mathcal{E}}(\vec{r}, t)=\frac{4 \pi}{c^{2}} \frac{\partial^{2}}{\partial t^{2}} \vec{P}(\vec{r}, t),
$$

where $c$ is the speed of light and $\vec{P}(\vec{r}, t)$ is the induced polarization in the target. Even though we use atomic units, we keep $c$ for the speed of light in Eq. (1). The factor of $4 \pi$ comes from the fact that $4 \pi \epsilon_{0}=1$ in atomic units.

To allow for an analytical solution, we impose a series of approximations. First, we assume that the electric field is a pulse propagating in the $x$ direction and that it is sufficiently wide in the transverse directions to neglect the $\frac{\partial^{2}}{\partial y^{2}}$ and $\frac{\partial^{2}}{\partial z^{2}}$ terms, i.e., we use the plane-wave approximation. A more restrictive approximation is that the target is very thin, such that we may neglect any propagation or phase-matching effects by treating the target as a point potential [14]. With these approximations, the Maxwell equation is reduced to

$$
\left(\frac{\partial^{2}}{\partial x^{2}}-\frac{1}{c^{2}} \frac{\partial^{2}}{\partial t^{2}}\right) \overrightarrow{\mathcal{E}}(x, t)=\frac{4 \pi}{c^{2}} \frac{\partial^{2}}{\partial t^{2}} \vec{P}(t) \delta(x),
$$

where $\delta(x)$ is a Dirac delta function. If we assume that the incoming field is a pulse propagating in the positive $x$ direction, the solution to this equation is

$$
\overrightarrow{\mathcal{E}}(x, t)=\overrightarrow{\mathcal{E}}_{\mathrm{in}}\left(t-\frac{x}{c}\right)+\overrightarrow{\mathcal{E}}_{\mathrm{gen}}(x, t),
$$

where

$$
\overrightarrow{\mathcal{E}}_{\mathrm{gen}}(x, t)=-\frac{2 \pi}{c}\left[\Theta(x) \frac{\partial}{\partial t} P\left(t-\frac{x}{c}\right)+\Theta(-x) \frac{\partial}{\partial t} P\left(t+\frac{x}{c}\right)\right]
$$

as can be seen by insertion. Here, $\Theta(x)$ is the Heaviside function, which is 1 for $x>0$ and 0 elsewhere.

The signal in the absorption experiment is the frequencyresolved electromagnetic field at a given position $x>0$, after the pulse has passed through the target. The spectrum of interest may be expressed as

$$
S(\omega) \propto\left|\int d t e^{i \omega t} \overrightarrow{\mathcal{E}}(x, t)\right|^{2} .
$$

If we introduce the expression from Eq. (3) into this expression for the signal, we find three terms

$$
S(\omega) \propto\left|\overrightarrow{\mathcal{E}}_{\text {in }}(\omega)\right|^{2}+2 \operatorname{Re}\left[\overrightarrow{\mathcal{E}}_{\text {in }}^{*}(\omega) \cdot \overrightarrow{\mathcal{E}}_{\text {gen }}(\omega)\right]+\left|\overrightarrow{\mathcal{E}}_{\text {gen }}(\omega)\right|^{2},
$$

where the Fourier transform of the incoming field is given by

$$
\overrightarrow{\mathcal{E}}_{\text {in }}(\omega)=\int d t e^{i \omega t} \overrightarrow{\mathcal{E}}_{\text {in }}(t)
$$

and where the Fourier transform of the generated field due to interaction with the polarization is given by

$$
\begin{aligned}
\overrightarrow{\mathcal{E}}_{\mathrm{gen}}(\omega) & =-\frac{2 \pi}{c} \int d t e^{i \omega t} \frac{\partial}{\partial t} \vec{P}(t) \\
& =\frac{2 \pi \omega i}{c} \int d t e^{i \omega t} \vec{P}(t) .
\end{aligned}
$$

Here we used the facts that the polarization is vanishing for $t \rightarrow \pm \infty$ due to relaxation and that the integration over all times allows us to make a coordinate transformation and eliminate the $x$ dependence.

From the above equations we see that, to determine the spectrum, it remains to find a way of calculating the polarization $\vec{P}(t)$. For a gas of atomic or molecular targets, the polarization may be written as

$$
\vec{P}(t)=n l\langle\vec{D}(t)\rangle
$$

where $n$ is the density of the target, $l$ is the length, and $\langle\vec{D}(t)\rangle$ is the expectation value of the atomic or molecular dipole moment. Here we calculate the expectation value of the dipole moment from a quantum-mechanical model describing the interaction of the incoming laser pulses with the target, assuming that the generated electric field is weaker and may be ignored in the calculation of the dipole moment.

In the present formulation it is clear that the signal in short-pulse, e.g., attosecond absorption spectroscopy, does not originate only from the instants of time when the electric field is nonvanishing. The integration over time, needed for the frequency-resolved signal, will pick up contributions over the entire lifetime of the polarization signal. For attosecond spectroscopy this latter lifetime will typically be determined by system-dependent relaxation processes like, e.g., radiative or Auger decay and will often be much longer than the duration of the pulses involved. The long temporal integration time is the reason why it is possible also in attosecond absorption spectroscopy to obtain a frequency resolution much better than the Fourier limit related to the duration of the pulse. The formulation in the time domain is natural for studying many dynamical problems. The typical experimental scenario is the following: A pump pulse initiates some dynamics that is probed by a probe pulse. The present formulation readily describes situations where the pump and the probe pulse overlap in time or when the probe pulse proceeds the pump pulse as was the case in Ref. [15]. See Refs. [16,17] for other related formulations of the theory.

The assumption of a thin gas was used in Eq. (2) to justify neglect of propagation effects. In a real experiment a denser gas would be advantageous to increase the probability for excitation and absorption. The main effect of propagation is absorption and dispersion. The number of free electrons is low due to low ionization probability, so we expect no significant dispersion effects. The effect of absorption in propagation is accounted for by Beer's law and does not affect the main findings and conclusions of the present work.

\section{ATOMIC RESPONSE}

The scheme we study is based on two very short (attosecond) pulses, one being used to ionize the target and the other 


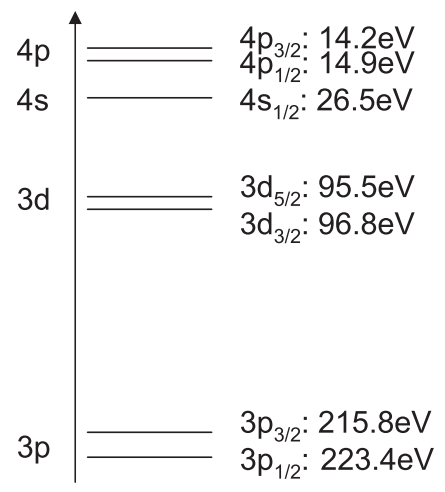

FIG. 1. Binding energies for the relevant states in the krypton atom. The energies are obtained from our calculations as discussed in Sec. IV.

to excite a core level electron into the hole created by the initial ionization. The signal we look for is the absorption of the second pulse at the frequency corresponding to the energy difference between the core and the hole level. We present two examples of attosecond absorption spectroscopy schemes used to study the hole formation and the hole evolution in krypton atoms. We refer to a state that is emptied by the pump pulse as a hole state and a state from which the probe pulse may promote an electron into the hole state as a core state. In the first example, the hole states are the $4 p$ states and the core states are the $3 d$ states, and in the other example, we consider a hole in the $4 s$ or $3 d$ states and probe it through the coupling to the $3 p$ core states. The relevant states in krypton are shown in Fig. 1, along with the calculated binding energy for each of the states (see Sec. IV for the details involved in these calculations.)

We consider the Hamiltonian

$$
H=H_{0}+V_{1}(t)+V_{2}\left(t-t_{0}\right),
$$

where $H_{0}$ denotes the field-free Hamiltonian, $V_{1}$ the interaction with the pump pulse, and $V_{2}$ the interaction with the probe pulse. Both laser pulses are linearly polarized along the $z$ axis. The laser-atom interaction is in the length-gauge dipole approximation, $V_{i}(t)=D_{z} \overrightarrow{\mathcal{E}}_{i}(t)(i=1,2)$, where $D_{z}$ is the dipole operator $D_{z}=\sum_{i=1}^{N} d_{z_{i}}=-\sum_{i=1}^{N} z_{i}$ with $N$ the number of electrons. The probe pulse $V_{2}\left(t-t_{0}\right)$ arrives with a variable delay $t_{0}$ relative to the pump pulse.

To calculate the time-dependent dipole moment entering Eq. (10), we make an essential state expansion of the manyelectron wave function

$$
\begin{aligned}
\Psi(t)= & c_{0}(t)|0\rangle+\sum_{a, r} c_{a}^{r}(t) e^{-i\left(E_{r}-E_{a}\right) t}\left|a^{-1}, r\right\rangle \\
& +\sum_{b, s} c_{b}^{s}(t) e^{-i\left(E_{s}-E_{b}\right) t}\left|b^{-1}, s\right\rangle,
\end{aligned}
$$

where $|0\rangle=|\{a b c \ldots n\}\rangle$ denotes the Slater determinant of orbitals for the ground state of the neutral atom, the sum over $a$ runs through all of the orbitals undergoing single-photon ionization from the first (pump) pulse, the sum over $r$ or $s$ runs through all of the continuum states accessible, and the sum over $b$ runs through all of the core orbitals that the second (probe) pulse excites into one of the hole states. In Eq. (12) we have suppressed the integral over the continua associated with $r$ and $s$ for notational convenience, and the state $\left|a^{-1}, r\right\rangle$ denotes that an electron in orbital $a$ has been excited to orbital $r$. Similar meaning is attributed to $\left|b^{-1}, s\right\rangle$. In this work, the formalism of second quantization is not needed; we just note that $\left|a^{-1}, r\right\rangle=\hat{c}_{r}^{\dagger} \hat{c}_{a}|0\rangle$ with $\hat{c}_{r}^{\dagger}$ and $\hat{c}_{a}$ creation and annihilation operators

We assume that the pulses are sufficiently weak that we may treat the interaction in lowest-order perturbation theory. The probability for creation of a hole state, due to the ionization by the pump pulse followed by excitation by the probe pulse, is proportional to $V_{1} V_{2}$, whereas ionization and excitation by the probe pulse is proportional to $V_{2}^{2}$. To be able to use the pumpprobe scheme, we need the first term to dominate, $V_{1}>V_{2}$, since the second part does not hold any information about the evolution that occurs during the time between the two pulses. Assuming that $V_{1}$ dominates, we neglect the ionization due to $V_{2}$ and this leads to the following equations for the coefficients in Eq. (12):

$$
\begin{aligned}
c_{0}(t)= & 1, \\
c_{a}^{r}(t)= & -i \int^{t} d t^{\prime} \overrightarrow{\mathcal{E}}_{1}\left(t^{\prime}\right) e^{i\left(E_{r}-E_{a}\right) t^{\prime}}\left\langle a^{-1}, r\left|D_{z}\right| 0\right\rangle, \\
c_{b}^{s}(t)= & -i \sum_{a, r} \int^{t} d t^{\prime} \overrightarrow{\mathcal{E}}_{2}\left(t^{\prime}-t_{0}\right) c_{a}^{r}\left(t^{\prime}\right) \\
& \times e^{i\left(E_{s}-E_{r}+E_{a}-E_{b}\right) t^{\prime}}\left\langle b^{-1}, s\left|D_{z}\right| a^{-1}, r\right\rangle .
\end{aligned}
$$

Since $D_{z}=\sum_{i=1}^{N} d_{z_{i}}$ is a sum of single-particle operators, the Slater-Condon rules allow us to reduce the many-electron matrix elements to single-electron integrals, $\left\langle a^{-1}, r\left|D_{z}\right| 0\right\rangle=$ $\left\langle r\left|d_{z}\right| a\right\rangle$ and $\left\langle b^{-1}, s\left|D_{z}\right| a^{-1}, r\right\rangle=\left\langle a\left|d_{z}\right| b\right\rangle \delta_{r, s}$. Equation (13) then simplifies to

$$
\begin{aligned}
& c_{0}(t)=1, \\
& c_{a}^{r}(t)=-i \int^{t} d t^{\prime} \overrightarrow{\mathcal{E}}_{1}\left(t^{\prime}\right) e^{i\left(E_{r}-E_{a}\right) t^{\prime}}\left\langle r\left|d_{z}\right| a\right\rangle, \\
& c_{b}^{s}(t)=-i \sum_{a} \int^{t} d t^{\prime} \overrightarrow{\mathcal{E}}_{2}\left(t^{\prime}-t_{0}\right) c_{a}^{s}\left(t^{\prime}\right) e^{i\left(E_{a}-E_{b}\right) t^{\prime}}\left\langle a\left|d_{z}\right| b\right\rangle .
\end{aligned}
$$

As discussed in Sec. I, the object of interest is the expectation value of the dipole moment,

$$
\left\langle D_{z}(t)\right\rangle=\sum_{a, b, r} c_{b}^{r}(t)^{*} c_{a}^{r}(t) e^{-i\left(E_{a}-E_{b}\right) t}\left\langle a\left|d_{z}\right| b\right\rangle+\text { c.c. },
$$

where we have included only the terms which include two bound states. The contributions to the dipole moment expectation value from the continuum states are assumed to give rise to no significant features, since the electron rapidly leaves the ions and the absorption is spectrally very broad.

After both pulses are over, the time evolution of the dipole moment is trivial as all of the expansion coefficients will no longer change. In our studies the duration of the attosecond pulses is shorter than the coherence lifetimes and the Auger lifetimes of the states involved so the major part of the contribution to the integral in Eq. (9) originates from 
times after the pulse is over. We evaluate the time integrals analytically using sine-squared envelopes,

$$
\overrightarrow{\mathcal{E}}_{i}(t)=\overrightarrow{\mathcal{E}}_{0, i} \sin ^{2}\left(\frac{\pi t}{\tau_{i}}\right) \cos \left(\omega_{i} t\right),
$$

for the two pulses with $\overrightarrow{\mathcal{E}}_{0, i}$ the peak electrical field strength, $\tau_{i}$ the duration of the pulse (with the full width at half maximum $\left.\tau_{\mathrm{FWHM}, i}=\tau_{i} / 2\right)$, and $\omega_{i}$ the central frequency.

The evaluation of the time-dependent part of the core hole coefficients requires consideration of all possible relative time orderings between the two pulses. The final expression is long and will not be presented here. Once the coefficients $c_{a}^{r}(\tau)$ and $c_{b}^{r}(\tau)$ are known, the full evaluation of the absorption spectrum is calculated from Eqs. (9) and (10),

$$
\overrightarrow{\mathcal{E}}_{\mathrm{gen}}(\omega)=\frac{2 \pi i \omega}{c} n l \vec{D}(\omega)
$$

with

$$
\begin{aligned}
\vec{D}(\omega)= & \int d t e^{i \omega t}\langle\vec{D}(t)\rangle \\
= & \sum_{a, b, r} c_{b}^{r}(\tau)^{*} c_{a}^{r}(\tau)\left\langle a\left|d_{z}\right| b\right\rangle \\
& \times \int_{t_{0}}^{\infty} d t e^{i \omega t} e^{-i\left(E_{a}-E_{b}\right) t} e^{-\frac{\Gamma_{a b}}{2}\left(t-t_{0}\right)} \\
= & i \sum_{a, b, r} c_{a}^{r}(\tau)^{*} c_{a}^{r}(\tau)\left\langle a\left|d_{z}\right| b\right\rangle \frac{e^{-i\left(E_{a}-E_{b}-\omega\right) t_{0}}}{E_{a}-E_{b}-i \frac{\Gamma_{a b}}{2}},
\end{aligned}
$$

where we have introduced $\Gamma_{a b}=\tau_{a b}^{-1}$, with $\tau_{a b}$ the lifetime of the coherence between the states $a$ and $b$. We approximate this coherence lifetime with the Auger lifetime of the shorter-living state. The complex conjugate part in Eq. (15) has been discarded, since it does not have any contribution in the frequency domain of interest for absorption; see Eq. (9).

If the hole state $a$ has a finite lifetime, we may describe this by introducing an imaginary part to the energy, as $E_{a} \rightarrow E_{a}-i \frac{\Gamma_{a}}{2}$. Assuming that this lifetime is much longer than the duration of the attosecond pulses, we can introduce it directly: At the arrival of the probe pulse, the amplitude $c_{a}^{r}(t)$ is decreased by $\exp \left(-\Gamma_{a} t_{0} / 2\right)$. Since $c_{b}^{r}$ is determined by $c_{a}^{s}$ [see Eq. (14)] it too is reduced by $\exp \left(-\Gamma_{a} t_{0} / 2\right)$. The final expression for the induced electric field then reads

$$
\overrightarrow{\mathcal{E}}_{\mathrm{gen}}(\omega)=-\frac{2 \pi \omega}{c} n l \sum_{a, b, r} c_{b}^{r *} c_{a}^{r}\left\langle a\left|d_{z}\right| b\right\rangle \frac{e^{-i\left(E_{a}-E_{b}-\omega-i \Gamma_{a}\right) t_{0}}}{E_{a}-E_{b}-i \frac{\Gamma_{b}}{2}},
$$

where we have suppressed the $\tau$ dependence on the coefficients to lighten the notation.

The result in Eq. (19) shows a series of absorption peaks, centered at the difference between the hole and the core states, $E_{a}-E_{b}$.

\section{CALCULATION OF ATOMIC PARAMETERS}

We use a fully relativistic approach for the calculation of atomic parameters: energies and transition rates. The method is outlined below and was presented in detail in Refs. [18-20]. It is based on relativistic many-body theory, combined with complex rotation to handle the coupling of a core hole with its Auger decay channels. This coupling can be strong and gives then also a significant energy level shift compared to the case when the coupling is ignored.

The calculations are performed in a radial basis defined through a one-particle Hamiltonian that includes a suitable spherically symmetric potential, here chosen as the HartreeFock potential (see more below). The finite-difference approximation is used to discretize the Hamiltonian on a radial grid. The distance between the grid points is exponentially increasing and reaches out to $\sim 20-30$ a.u. Diagonalization of the Hamiltonian matrix gives a radial basis set that is complete on the grid [21]. Typically 200 basis functions are obtained for each $\ell_{j}$ symmetry. The many-body calculation, i.e., the systematic correction of the first one-particle approximation toward the full many-particle solution, is carried through with this radial basis. All angular integrations are performed analytically using angular momentum algebra.

The wave functions used for the calculation of the atomic response discussed in Sec. III are constructed with the atomic parameters as input.

\section{A. Binding energies}

The binding energy of electron $a$ in atom $A$ is the difference between the the energy of the ion $A^{+}$(where $a$ has been removed) and the the energy of the atom. The first approximation to this quantity is the Hartree-Fock energy of orbital $a$ in the atom. Within a relativistic formulation we define the orbitals as eigenstates to the one-particle Hamiltonian

$$
h_{\mathrm{DF}}=c \boldsymbol{\alpha} \cdot \mathbf{p}+(\beta-1) m c^{2}-\frac{Z}{r}+V_{\mathrm{DF}},
$$

where $\alpha$, and $\beta$ are the Dirac matrices. The Hamiltonian in (20) is labeled Dirac-Fock to emphasize that the Hartree-Fock method is implemented in the framework of the Dirac equation. The potential $V_{\mathrm{DF}}$ is, as in nonrelativistic calculations, defined through its action on the occupied orbitals,

$$
V_{\mathrm{DF}}|a\rangle=\sum_{b}^{\text {core }}\left\langle b\left|V_{12}\right| b\right\rangle|a\rangle-\left\langle b\left|V_{12}\right| a\right\rangle|b\rangle
$$

where the sum over $b$ runs over all occupied orbitals and $V_{12}$ denotes the electron-electron interaction. For more details, see, e.g., [22]. For a closed-shell system, like krypton considered here, $V_{\mathrm{DF}}$ is spherically symmetric. It includes the, usually dominating, Coulomb interaction

$$
\frac{1}{r_{12}}
$$

but may also be generalized to include corrections due to the finite speed of the electromagnetic interaction, as well as magnetic contributions present due to the electron spin, through the Breit operator [23],

$$
-\frac{1}{2}\left(\frac{\boldsymbol{\alpha}_{1} \cdot \boldsymbol{\alpha}_{2}}{r_{12}}+\frac{\left(\boldsymbol{\alpha}_{1} \cdot \mathbf{r}_{\mathbf{1 2}}\right)\left(\boldsymbol{\alpha}_{2} \cdot \mathbf{r}_{\mathbf{1 2}}\right)}{r_{12}^{3}}\right),
$$

where the indices refer to particles 1 and 2 in the interacting electron pair. Here all contributions to the energy up to the order $\alpha^{2}$ a.u. are included. 
TABLE I. Krypton binding energies. The results are given in $\mathrm{eV}$.

\begin{tabular}{|c|c|c|c|c|c|c|c|}
\hline & $3 p_{1 / 2}$ & $3 p_{3 / 2}$ & $3 d_{3 / 2}$ & $3 d_{5 / 2}$ & $4 s_{1 / 2}$ & $4 p_{1 / 2}$ & $4 p_{3 / 2}$ \\
\hline Dirac-Fock & 234.56 & 226.20 & 102.79 & 101.41 & 32.32 & 14.74 & 14.00 \\
\hline Relaxation & -8.42 & -8.33 & -8.47 & -8.47 & -1.40 & -1.04 & -1.00 \\
\hline$\Delta$ Dirac-Fock-Breit & -0.33 & -0.18 & -0.02 & 0.04 & -0.01 & -0.02 & 0.00 \\
\hline Correlation & 2.54 & 2.52 & 4.21 & 4.20 & 1.73 & 2.23 & 2.20 \\
\hline Auger type admixtures & -4.92 & -4.40 & -1.74 & -1.74 & -6.11 & -1.05 & -1.01 \\
\hline \multirow[t]{2}{*}{ Sum } & 223.43 & 215.82 & 96.78 & 95.45 & 26.53 & 14.86 & 14.18 \\
\hline & $222.39^{\mathrm{a}}$ & $214.79^{\mathrm{a}}$ & $95.44^{\mathrm{a}}$ & $94.19^{\mathrm{a}}$ & $27.51^{\mathrm{b}}$ & $14.67^{\mathrm{b}}$ & $14.00^{\mathrm{b}}$ \\
\hline
\end{tabular}

${ }^{\text {a Reference [26]. }}$

${ }^{\mathrm{b}}$ Reference [27].

The use of Eqs. (22) and (23) implies that we work in the so-called Coulomb gauge. Other choices are possible, but it has been shown that the Coulomb gauge is the most practical choice for modestly charged systems [24,25].

As stated in Koopmans' theorem the orbital energy, i.e., the eigenvalue of (20), would be the binding energy if all the other electrons were unaffected when one electron is removed from the system. As can be seen in Table I the bulk contribution to the binding energy is indeed given by the Dirac-Fock orbital energy, with some additional contribution, at least for the deepest core holes, when also the Breit interaction is included (given separately on the third row in the table). The next step is to account for the adjustment of the other electrons. For this we have to go beyond the Hartree-Fock approximation and consider the remaining electron-electron interaction, here restricted to the remaining Coulomb interaction. We divide the contributions mixed in through this interaction into different categories. First, the decreased screening of the nuclear charge leads to a contraction of the ionic wave function compared to the atom. This relaxation will naturally decrease the binding energy. Starting with the Slater determinant after ionization of one electron from orbital $a$,

$$
\left|a^{-1}\right\rangle
$$

we account for relaxation through admixtures of configurations where one orbital is replaced by a previously unoccupied orbital. These single excitations can be schematically written as

$$
\sum_{r}^{\text {exc }} \sum_{b}^{\text {core }}\left|\left\{a^{-1} b^{-1} r\right\}\right\rangle,
$$

where the curly brackets denote antisymmetrization. Through an iterative procedure these admixtures are included to all orders [18]. In addition there are true many-electron effects. We list separately the admixture of double excitations into the wave function (treated to second order in the remaining Coulomb interaction and labeled "Correlation" in Table I), and finally admixtures of the type

$$
\sum_{r}^{\text {exc }} \sum_{b c}^{\text {core }}\left|\left\{b^{-1} c^{-1} r\right\}\right\rangle \text {. }
$$

In the last contribution the initial hole in $a$ has been refilled by a previously occupied orbital, while another orbital has been excited. This corresponds to a transition $b c \rightarrow a r$, i.e., to an Auger-type transition. Such contributions to the wave function exist even if the transition is not energetically allowed. When the energy of the $a$ hole configuration is close to that of the $b c$ hole configuration, this contribution can be significant and may also vary strongly from shell to shell (or from element to element). This is in contrast to the other contributions listed in Table I, which generally are rather similar for nearby shells. In the present case we see a strong contribution to the $4 \mathrm{~s}$ hole from $4 p^{2}$ hole Auger-type admixtures, although Auger transitions are not allowed energetically for a $4 s$ hole. It is interesting to note that in the lowest-order approximation one would expect the $4 s$ hole to be subject to Auger decay; the Dirac-Fock orbital energy for $4 s$ is larger than twice the $4 p_{j}$ energy. It is many-body effects that stabilize the state against electron emission. We discuss the calculations of these contributions more below.

\section{B. Auger rates}

Holes in $n=3$ and below can decay by electron emission: $A_{j m}^{+} \rightarrow\left\{A^{2+}+\varepsilon\right\}_{j m}$, where the ejected electron has the energy $\varepsilon=E_{A^{+}}-E_{A^{2+}}$. With the method of complex scaling the energy half-width is calculated as the imaginary part of a now complex energy eigenvalue. It is illustrative to study the method in second-order perturbation theory. Admixtures like those listed in Eq. (26) will then contribute to the $a$ hole state with the energy

$$
\Delta E=\sum_{r}^{\text {ex }} \sum_{b c}^{\text {core }} \frac{\left\langle b c\left|r_{12}^{-1}\right| a r\right\rangle\left\langle a r\left|r_{12}^{-1}\right| b c\right\rangle}{\varepsilon_{b}+\varepsilon_{c}-\varepsilon_{a}-\varepsilon_{r}},
$$

where the pole contribution (at $\varepsilon_{r}=\varepsilon_{b}+\varepsilon_{c}-\varepsilon_{a}$ for $\varepsilon_{b}+$ $\varepsilon_{c}-\varepsilon_{a}>0$ ) gives the Auger half-life and the principal value integral gives a level shift. With complex scaling $r \rightarrow r e^{i \theta}$, the continuum electron $r$ is described by a pseudocontinuum with complex energies, with approximately $\arg \left(\varepsilon_{r}\right)=-2 \theta$. Thereby the integration over continuum energies is performed over a contour in the complex plane, the poles are no longer on the path of integration, and standard numerical schemes gives stable results. A description of the combination of complex rotation and many-body perturbation theory in an all-order scheme can be found in Ref. [28]. For deep core holes second-order perturbation theory generally gives good results, but for the holes in $n=3,4$ considered here it is necessary to go to higher orders. Then the different Auger channels, energetically allowed or not, will also be coupled. With an iterative scheme all contributions, such as those listed in 
TABLE II. Auger transition rates in krypton obtained by the method described in Sec. IV B. Results are given in $\mathrm{fs}^{-1}$.

\begin{tabular}{lccc}
\hline \hline $3 p_{1 / 2}$ & $3 p_{3 / 2}$ & $3 d_{3 / 2}$ & $3 d_{5 / 2}$ \\
\hline 1.52 & 1.64 & 0.075 & 0.078 \\
\hline \hline
\end{tabular}

Eq. (26), can be mixed and included to all orders. However, for hole states with a strong coupling to one or several Auger channels, e.g., when $\varepsilon_{b}+\varepsilon_{c} \approx \varepsilon_{a}$, the iterative scheme often fails to converge. In those cases we identify the most important channels in second order, Eq. (27), and account for the mixing through a configuration-interaction type of calculation. The Auger lifetimes are given in Table II. For the $3 p$ holes the final Auger rate is $\sim 50 \%$ smaller than the second-order results, while for the $3 d$ holes it is $\sim 25 \%$ smaller.

\section{Radiative transitions between bound states}

As discussed in Sec. III, the probe pulse can promote a core electron into the hole created by the pump pulse and the amplitude for this process is governed by dipole matrix elements: $\left\langle a\left|d_{z}\right| b\right\rangle=-\langle a|z| b\rangle$; cf. Eq. (15). According to the Wigner-Eckart theorem such matrix elements can be written in terms of $3 j$ symbols and reduced matrix elements as

$$
\begin{aligned}
& \left\langle\gamma_{a} j_{a} m_{a}|z| \gamma_{c} j_{b} m_{b}\right\rangle \\
& =(-1)^{j-m}\left(\begin{array}{ccc}
j_{a} & 1 & j_{b} \\
-m_{a} & 0 & m_{b}
\end{array}\right)\left\langle\gamma_{a} j_{a}|| \mathbf{r} \| \gamma_{b} j_{b}\right\rangle,
\end{aligned}
$$

where $j$ and $m$ are the quantum numbers of the involved orbitals and also denote the symmetry of the particular hole state. The label $\gamma$ indicates other quantum numbers specifying the state. The reduced matrix element contains all information about the radial overlap and is independent of the $m$ values. We list the reduced matrix elements, $\left\langle\gamma_{a} j_{a}|| \mathbf{r}|| \gamma_{b} j_{b}\right\rangle$, as calculated within the Dirac-Fock approximation, for all the involved orbitals in Table III.

It is also interesting to use the information about radiative transitions between bound states to calculate the spontaneous radiative decay of the holes; see Table IV. Compared to the Auger rates these rates are three to four orders of magnitude lower.

TABLE III. Reduced transition matrix elements [cf. Eq. (28)] which determine the transition rates between different core hole states in krypton. The results are given in atomic units.

\begin{tabular}{lr}
\hline \hline$\left\langle 3 d_{3 / 2}|| \mathbf{r}|| 3 p_{1 / 2}\right\rangle$ & \\
$\left\langle 3 d_{3 / 2}|| \mathbf{r} \| 3 p_{3 / 2}\right\rangle$ & -0.601 \\
$\left\langle 3 d_{5 / 2}|| \mathbf{r}|| 3 p_{3 / 2}\right\rangle$ & 0.271 \\
$\left\langle 4 s_{1 / 2}|| \mathbf{r}|| 3 p_{1 / 2}\right\rangle$ & -0.817 \\
$\left\langle 4 s_{1 / 2}|| \mathbf{r}|| 3 p_{3 / 2}\right\rangle$ & -0.118 \\
$\left\langle 4 p_{1 / 2}|| \mathbf{r}|| 3 d_{3 / 2}\right\rangle$ & -0.183 \\
$\left\langle 4 p_{3 / 2}|| \mathbf{r}|| 3 d_{3 / 2}\right\rangle$ & -0.258 \\
$\left\langle 4 p_{3 / 2}|| \mathbf{r} \| 3 d_{5 / 2}\right\rangle$ & -0.110 \\
$\left\langle 4 p_{1 / 2}|| \mathbf{r} \| 4 s_{1 / 2}\right\rangle$ & -0.337 \\
$\left\langle 4 p_{3 / 2}|| \mathbf{r}|| 4 s_{1 / 2}\right\rangle$ & 1.378 \\
\hline \hline
\end{tabular}

TABLE IV. Spontaneous radiative decay rates for core holes. Results are given in $\mathrm{ps}^{-1}$.

\begin{tabular}{lllll}
\hline \hline $3 p_{1 / 2}$ & $3 p_{3 / 2}$ & $3 d_{3 / 2}$ & $3 d_{5 / 2}$ & $4 s_{1 / 2}$ \\
\hline 0.501 & 0.446 & 0.014 & 0.013 & 0.018 \\
\hline \hline
\end{tabular}

\section{Radiative transitions between bound and continuum states}

To obtain the time-dependent wave function we need also radiative transitions between bound and continuum states; cf. Eq. (14). The bound-state orbitals are obtained as described above. For the continuum, we solve the nonrelativistic Schrödinger equation with a local potential constructed from the relativistic Dirac-Fock electron density. The direct part of the electronic potential is just the solution of Poisson's equation. To ensure the correct asymptotic Coulomb potential, an exchange-correlation potential is added as described in Ref. [29]. The continuum states were solved in radial box of range 200 a.u., and hence we have a discretized representation of the continuum. Note that in the absorption experiments, the electrons are not measured, and hence the theory is insensitive to the scattering phase shifts of the outgoing electrons.

Figures 2 and 3 show the radial dipole matrix elements of the reduced wave functions for the initial- and final-state orbitals, i.e.,

$$
\int_{0}^{\infty} d r \phi_{r}(r) r \phi_{a}(r) .
$$

For a given electron continuum energy, the matrix elements entering the calculations, $\left\langle\gamma_{r} j_{r} m_{r}|z| \gamma_{a} j_{a} m_{a}\right\rangle$, are obtained from the the radial dipole matrix elements by multiplication by appropriate angular factors.

\section{RESULTS AND DISCUSSION}

With the energies, rates, and dipole matrix elements determined by the methods described in Sec. IV, we are ready to use

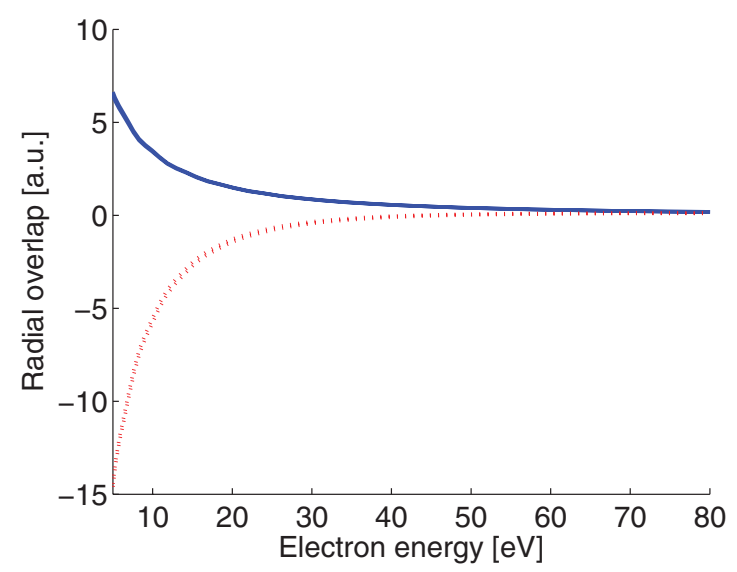

FIG. 2. (Color online) The radial dipole matrix element, Eq. (30), between the $4 p$ states in krypton and the $s$-wave continuum (full, blue) or the $d$-wave continuum (dotted, red). The overlaps are calculated for the $4 p_{j=1 / 2}$ and $4 p_{j=3 / 2}$ states. The radial difference in these latter states is very small and the difference in the radial overlaps cannot be seen on the scale of the figure. 


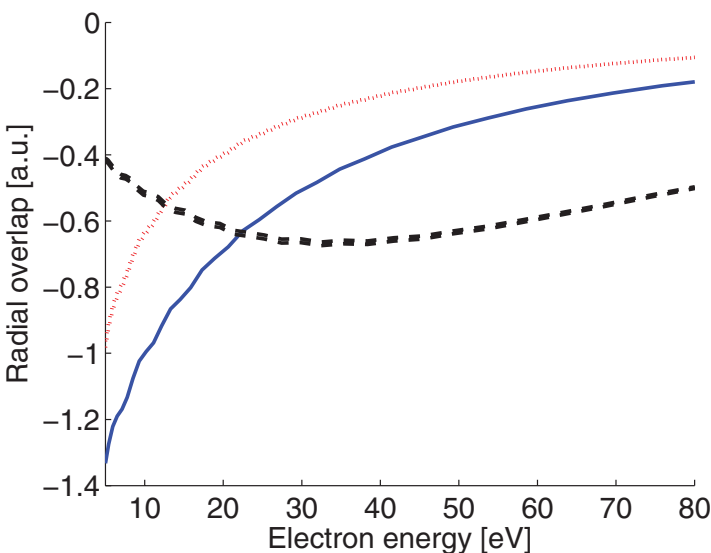

FIG. 3. (Color online) The radial dipole matrix element, Eq. (30), between the $4 s$ state in krypton and the $p$-wave continuum (full, blue) and the overlap between the $3 d$ states and the $p$-wave continuum (dotted, red) or the $f$-wave continuum (dashed, black). As in Fig. 2, the difference in the radial overlaps for the different $j$ states cannot be see in the figure.

the formalism of Secs. II and III to study illustrative examples of attosecond photoabsorption spectroscopy in krypton.

We calculate the absorption spectrum for two different sets of pump and probe pulses. First, we consider ionization from the $4 p$ valence band (see Fig. 1), followed by excitation from the $3 d$ to the $4 p$ level. This scenario is very close to what has already been measured in Ref. [6], with the difference that in Ref. [6] strong-field ionization was used to form the $4 p$ holes. Here, we consider an attosecond pulse for both the ionization and the subsequent excitation, allowing a more controlled study of the hole formation process.

For the second set of pump and probe pulses, we consider attosecond pulses with higher photon energies, such that the initial ionization may take place from the $4 s$ or $3 d$ levels and the subsequent excitation promotes electrons from the $3 p$ level to the holes. This allows us to simultaneously probe the hole formation in the $4 s$ and the $3 d$ levels, as well as to study the decay of the $3 d$ hole, which is above the threshold for Auger decay.

\section{A. Ionization from $4 p$ : Coupling $3 d$ and $4 p$}

To create the hole in the $4 p$ valence shell, we use a pump pulse with a central frequency of $\omega_{1}=50 \mathrm{eV}$ and a duration $\tau_{1}=450$ as. The probe pulse is centered at $\omega_{2}=81 \mathrm{eV}$ appropriate for the coupling of the $3 d$ and $4 p$ states and its duration is the same as that of the pump pulse. The duration of the pulses ensures that they are so spectrally narrow that the pump pulse cannot itself drive the $3 d-4 p$ transition. The strength of the pulses gives an overall scaling of the strength of the signal, and the intensity is kept such that both pulses lead to interactions that are in the perturbative regime. Furthermore, we keep the intensity of the second pulse such that it induces only bound-bound transitions, and ionization due to this pulse may be ignored. The spectral profiles of the two pulses are shown in Fig. 4.

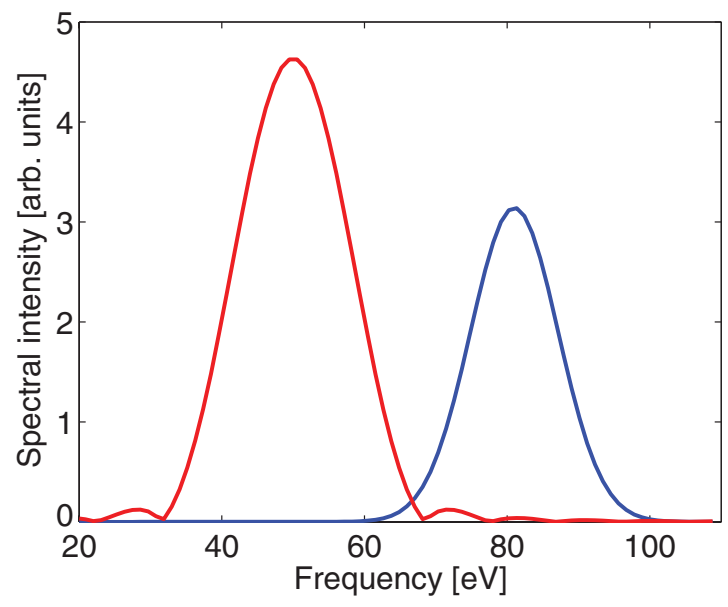

FIG. 4. (Color online) Spectral profiles of the two pulses (in arbitrary units). The photon energy of the pump pulse, centered at $50 \mathrm{eV}$, is insufficient to drive the transitions from the $3 d$ core levels to the $4 p$ valence holes, which are just above $80 \mathrm{eV}$. The intensity of the probe pulse, centered at $81 \mathrm{eV}$, is insufficient to cause ionization of the system.

We construct the signal from Eq. (6) by subtracting the contribution from the incoming field and by considering only the dominant term, i.e., the spectrum reads

$$
S\left(t_{0}, \omega\right) \propto \operatorname{Re}\left[\overrightarrow{\mathcal{E}}_{\text {in }}^{*}(\omega) \cdot \overrightarrow{\mathcal{E}}_{\text {gen }}(\omega)\right],
$$

where $t_{0}$ denotes the relative delay between the pulses. In a measurement, the contribution to the spectrum from the incoming field may be subtracted by recording the absorption spectrum when the probe pulse precedes the pump pulse by a sufficiently long time that there is no overlap in the signals from the two pulses. The generated field is calculated from Eq. (19). In the spectra shown, the coefficients are calculated for the sine-squared envelope of the field, while the field is assumed Gaussian in the evaluation of the Fourier transform to avoid any artificial tails extended to frequencies far from the central one. The spectrum is shown in Fig. 5 for different

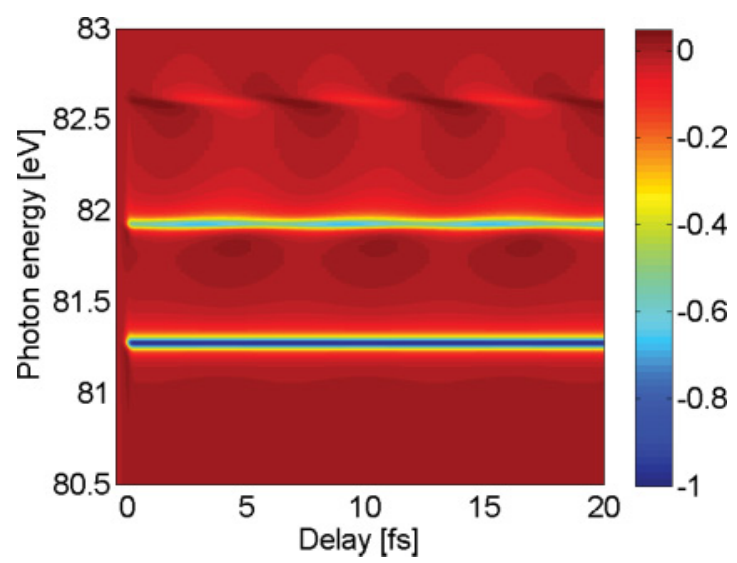

FIG. 5. (Color online) Normalized absorption spectrum for holes in the $4 p$ valence shell. The spectrum shows three lines at $81.3,81.9$, and $82.6 \mathrm{eV}$ corresponding to the $3 d_{5 / 2} \rightarrow 4 p_{3 / 2}, 3 d_{3 / 2} \rightarrow 4 p_{1 / 2}$, and $3 d_{3 / 2} \rightarrow 4 p_{3 / 2}$ transitions. 


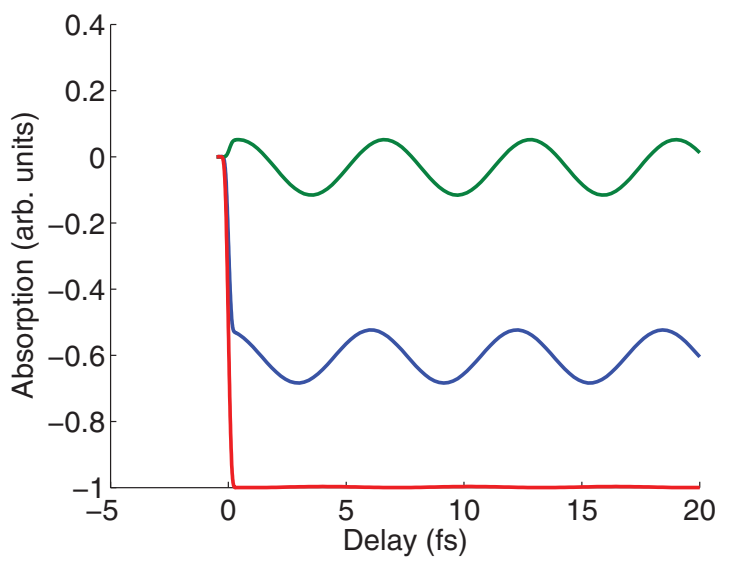

FIG. 6. (Color online) The absorption signal versus the delay between the pulses. The curves are (from strongest signal to weakest) $3 d_{5 / 2} \rightarrow 4 p_{3 / 2}$ (lowest curve, red), $3 d_{3 / 2} \rightarrow 4 p_{1 / 2}$ (middle curve, blue), and $3 d_{3 / 2} \rightarrow 4 p_{3 / 2}$ (highest curve, green).

values of the time delay of the probe pulse with respect to the pump pulse.

In the spectrum, three lines are seen corresponding to the $3 d$ to $4 p$ transitions. In Fig. 6 the signal at the center of the three lines is shown. The $3 d_{5 / 2} \rightarrow 4 p_{3 / 2}$ line (lowest, red) shows no oscillations, since a $3 d_{5 / 2}$ hole can be created through only one path: if the electron is promoted to an empty $4 p_{3 / 2}$ orbital.

Turning to the absorption line at $81.9 \mathrm{eV}$ corresponding to the $3 d_{3 / 2} \rightarrow 4 p_{1 / 2}$ transition, we find that this is weaker and that it shows oscillations with a period of $6.2 \mathrm{fs}$, corresponding to an energy difference $\Delta E=2 \pi / T=0.68 \mathrm{eV}$, which is exactly the difference between the two $4 p$ states. A hole in $3 d_{3 / 2}$ can indeed be created through two different channels, either via an intermediate $4 p_{1 / 2}$ hole or via a $4 p_{3 / 2}$ hole; cf. Eqs. (14). Because the time evolution of the intermediate states is different, these two channels interfere constructively or destructively depending on the delay between the two pulses, which is the reason for the oscillations. For each $m_{j}$ we have

$$
\begin{aligned}
c_{3 d_{3 / 2, m_{j}}}^{r}= & -\left\langle 4 p_{1 / 2}^{m_{j}}\left|d_{z}\right| 3 d_{3 / 2}^{m_{j}}\right\rangle \int d t^{\prime} \overrightarrow{\mathcal{E}}_{2}\left(t^{\prime}-t_{0}\right) e^{i\left(E_{4 p_{1 / 2}}-E_{3 d_{3 / 2}}\right) t^{\prime}} \\
& \times\left\langle r\left|d_{z}\right| 4 p_{1 / 2}^{m_{j}}\right\rangle \int d t^{\prime} \overrightarrow{\mathcal{E}}_{1}\left(t^{\prime}\right) e^{i\left(E_{r}-E_{4 p_{1 / 2}}\right) t^{\prime}} \\
& -\left\langle 4 p_{3 / 2}^{m_{j}}\left|d_{z}\right| 3 d_{3 / 2}^{m_{j}}\right\rangle \int d t^{\prime} \overrightarrow{\mathcal{E}}_{2}\left(t^{\prime}-t_{0}\right) e^{i\left(E_{4 p_{3 / 2}}-E_{3 d_{3 / 2}}\right) t^{\prime}} \\
& \times\left\langle r\left|d_{z}\right| 4 p_{3 / 2}^{m_{j}}\right\rangle \int d t^{\prime} \overrightarrow{\mathcal{E}}_{1}\left(t^{\prime}\right) e^{i\left(E_{r}-E_{4 p_{3 / 2}}\right) t^{\prime}}
\end{aligned}
$$

The oscillations in Fig. 6 are due to the second pulse being centered around $t_{0}$ and the integral acquiring a phase $e^{i\left(E_{4 p_{j}}-E_{3 d_{3 / 2}}\right) t_{0}}$ which is different for the two terms in Eq. (31). As in the experiment with strong-field ionization [6], in this perturbative regime, the pump pulse sets up a coherent superposition in the $4 p$ valence shell and this superposition is interfering either constructively or destructively as a $3 d$ core electron is excited to the valence hole. The dominant term goes via the $4 p_{1 / 2}$ state and gives rise to the constant background in the signal, while the oscillations are due to the pathway involving the $4 p_{3 / 2}$ state.
For the weakest of the absorption lines, the $3 d_{3 / 2} \rightarrow 4 p_{3 / 2}$ line at $82.6 \mathrm{eV}$, the oscillations are still due to the two different pathways to the $3 d_{3 / 2}$ state and still the pathway via $4 p_{1 / 2}$ is dominant. Again, since the time evolution of the two $4 p$ states is different, the pathway via the $4 p_{1 / 2}$ state leads to oscillations and these are now greater than the constant signal originating from the $4 p_{3 / 2}$ pathway. This means that the generated field adds constructively to the incoming field.

Absorption spectroscopy with attosecond pumpattosecond probe pulses allows us to study the hole formation on a very short time scale, by comparing the buildup of the absorption signal as the pulses overlap. Zooming in on only the region where the two pulses overlap, we are able to temporally resolve the hole formation on an attosecond time scale. This is shown in Fig. 7. Since the $4 p$ hole has to be established before a $3 d$ electron can absorb a photon and transfer to the $4 p$ orbital, the absorption increases when a larger and larger fraction of the pump pulse comes before the probe pulse. From the figure we see that the absorption stabilizes starting at about a delay of 0.2 fs or higher. This corresponds to the situation in Fig. 8. When the
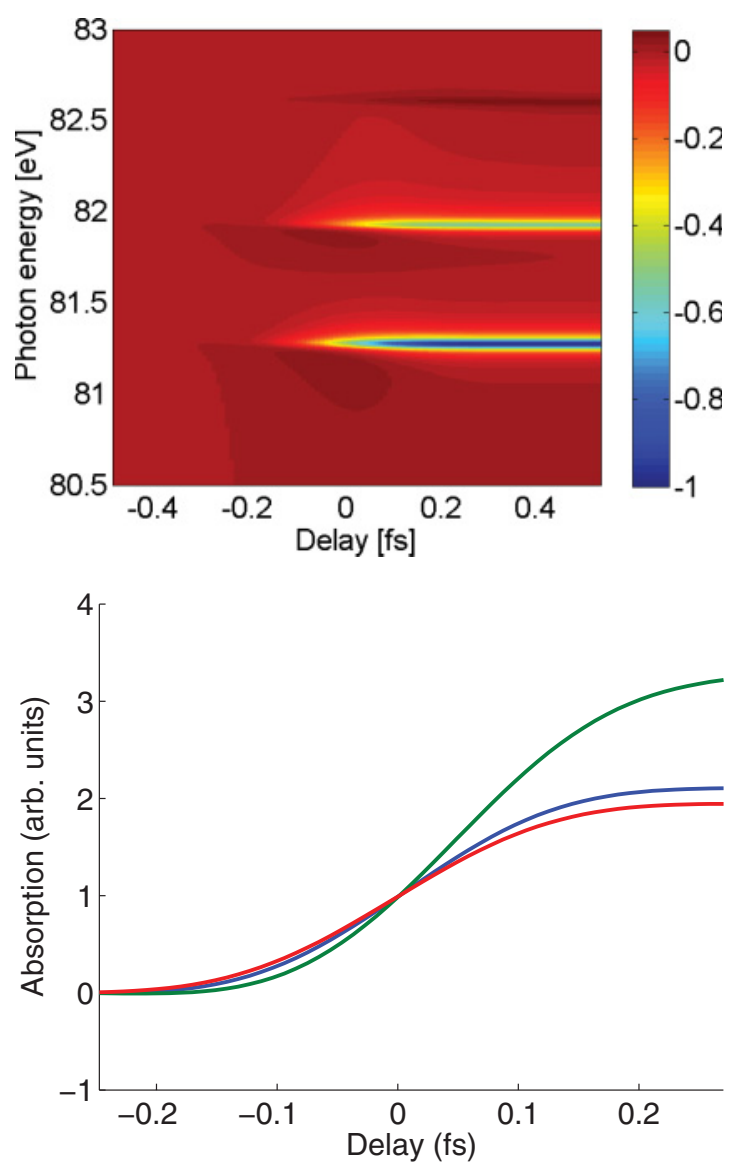

FIG. 7. (Color online) Focusing only on the times when the two pulses overlap, we may resolve the buildup of the holes on an attosecond time scale. The upper panel shows the absorption spectrum and the lower panel shows the absorption for the three lines, normalized at $t_{0}=0$. The curves are $3 d_{5 / 2} \rightarrow 4 p_{3 / 2}$ (lowest curve at a delay of $0.2 \mathrm{fs}$, red), $3 d_{3 / 2} \rightarrow 4 p_{1 / 2}$ (middle curve at a delay of $0.2 \mathrm{fs}$, blue), and $3 d_{3 / 2} \rightarrow 4 p_{3 / 2}$ (highest curve at a delay of 0.2 fs, green). 


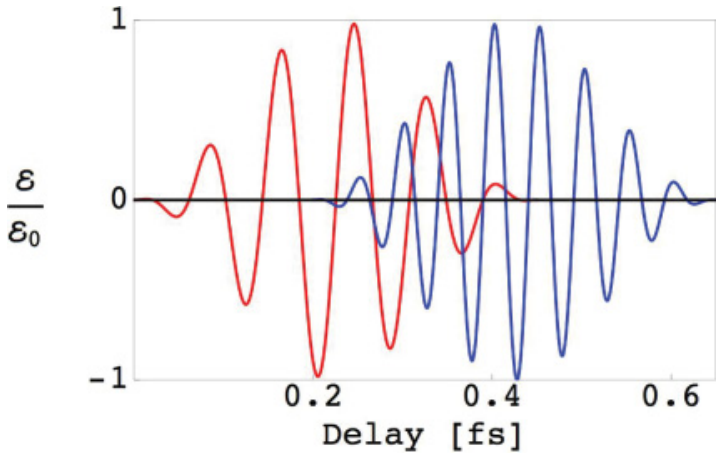

FIG. 8. (Color online) The absorption in Fig. 7 stabilizes at about a delay of $0.2 \mathrm{fs}$. This corresponds to the situation shown above.

time delay is around $0.45 \mathrm{fs}$ there is no more overlap between the two pulses.

In our model, we include no relaxation effects in the ion, treating the electrons as independent within the Hartree-Fock approximation. Hence, what we see as the buildup of the holes is mostly determined by the temporal profile of the two pulses involved. If many-electron effects are at play and give rise to a delay in the hole formation on the attosecond time scale, the method proposed here might be able to reveal differences in the formation time by showing a delay between the rise of the absorption curves or short-time oscillations in the absorption [30,31]. The differences seen in the lower panel in Fig. 7 are due to the interference between the different population channels; the reason is the same as that for the oscillations in Fig. 6.

\section{B. Ionization from $3 d$ : Coupling $3 p$ and $3 d$}

Perhaps more interesting is to study the case where the pump pulse removes an inner-shell electron and the probe pulse then promotes a core electron to the hole. As an example, we propose using an attosecond pulse to ionize an electron from the krypton $3 d$ shell and then promote a $3 p$ electron into the $3 d$ hole (see Fig. 1). In this example, we further make the probe pulse spectrally broad, such that it may also drive the transition from the $3 p$ to the $4 s$ state.

We calculate the absorption spectrum for a pump pulse centered at $\omega_{1}=100 \mathrm{eV}$ and with a slightly longer duration of $\tau_{1}=750$ as, such that it is spectrally narrower and does not populate the Rydberg states in the krypton atom. The probe pulse is centered at $\omega_{2}=160 \mathrm{eV}$ and has a duration of $\tau_{2}=$ 100 as, which makes is spectrally sufficiently broad to drive both the $3 p$ to $3 d$ and the $3 p$ to $4 s$ transitions. Again, we have used a sine-squared envelope for the calculations of the atomic coefficients in Eq. (14), but a Gaussian pulse when calculating the spectra.

In Fig. 9 the absorption spectra as well as the absorption at the center of the lines are shown. Turning first to the $3 p$ to $3 d$ lines (first and second panels), we see that the finite lifetime of the $3 d$ state now leads to a decrease of the signal as the probe pulse is delayed from the pump pulse. Similarly to the example discussed in Sec. V A, there are two different pathways to create a hole in the $3 p_{3 / 2}$ state, leading to interference between the channels and oscillations in the signal. This gives access to a direct time-domain measurement of both the lifetime of
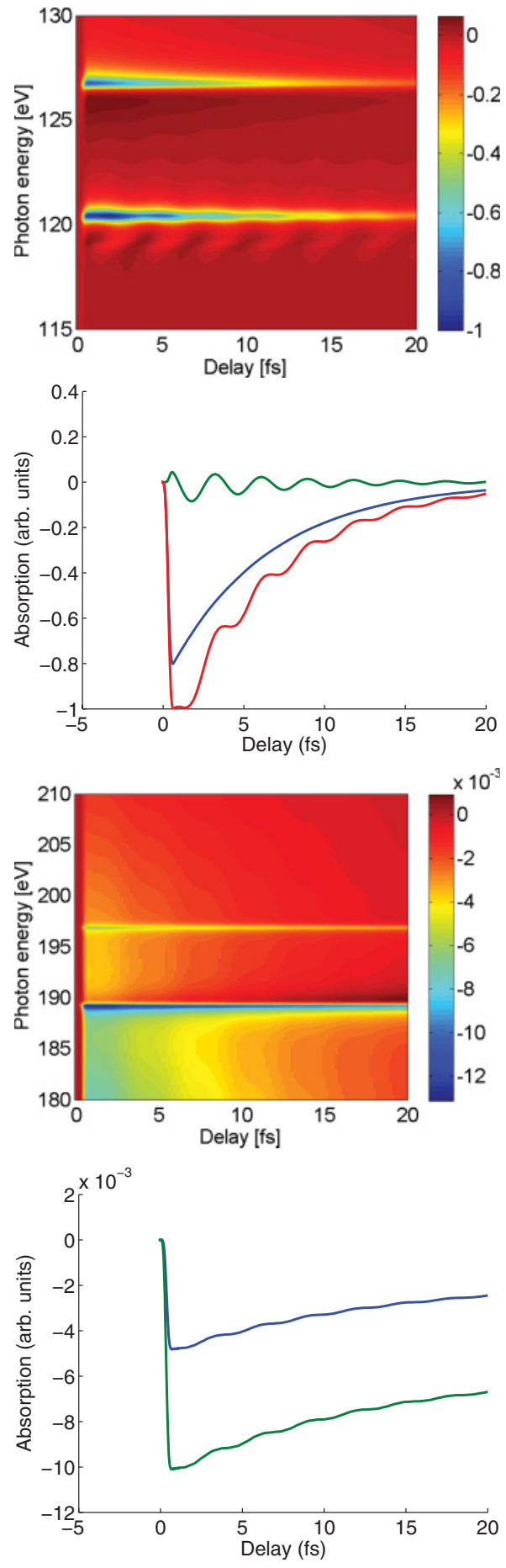

FIG. 9. (Color online) Absorption spectra calculated for the set of pulses tuned to drive transitions from the $3 p$ level. The spectra in the first and the second panels show the lines corresponding to the $3 d$ levels and the third and the fourth panels show the spectra for the $4 s$ level. The first and third panels show the full spectra. The second and the fourth panels show the intersections at the center of the lines. In the first and second panels the lines are $126.6 \mathrm{eV}, 3 p_{1 / 2}-3 d_{3 / 2}$ (middle curve, blue); $120.3 \mathrm{eV}, 3 p_{3 / 2}-3 d_{5 / 2}$ (lowest curve, red); $119 \mathrm{eV}, 3 p_{3 / 2}-3 d_{3 / 2}$ (highest curve, green). In the third and fourth panels the lines are 189.e eV, $3 p_{1 / 2}-4 s_{1 / 2}$ (upper curve, blue), $3 p_{3 / 2}-4 s_{1 / 2}$ (lower curve, blue). Notice that the set of parameters used implies that the absorption at the $3 p$ to $4 s$ lines is two orders of magnitude weaker than that at the $3 p$ to $3 d$ lines. 
the $3 d$ levels and the lifetime of the coherence in the $3 d$ levels, through the decay of the signal and the decay of the oscillations, which might be on a different time scale (though they are not in our model). The lifetime of the $3 d$ states is 13.3 fs for the $3 d_{3 / 2}$ state and 12.8 fs for the $3 d_{5 / 2}$.

The $4 s$ state (third and fourth panels in Fig. 9) is below the Auger threshold and is expected to live very long, the lifetime due to spontaneous radiative decay is $\sim 500 \mathrm{ps}$. With the choice of pump pulse aimed at coupling primarily the $3 p$ and $3 d$ levels, the $4 s$ level is, however, only weakly populated and hence the signal is weak. The decay, i.e., the decrease of the absolute signal in the figure, is the tail of the $3 p$ to $3 d$ transitions, which extend even to these much higher energies. The signal from the $4 s$ level is at about $1 \%$ of the signal from the major lines.

\section{CONCLUSION AND OUTLOOK}

We have derived a theory for attosecond pump-attosecond probe spectroscopy. We illustrated the theory by absorption spectroscopy in krypton. The light intensity and photon energy is such that the interaction between the attosecond pulses and the atomic target can be treated perturbatively. This means that we can use accurate time-independent many-electron structure theory for the extraction of the atomic parameters entering the model. We considered two examples. In the first example, we emptied a valence shell and coupled an inner shell to the hole. By varying the delay between the pump and the probe, we saw how the absorption signal varied on a subfemtosecond time scale. In the second example, an inner shell was emptied by the pump pulse and the probe coupled an even more tightly bound state to that hole. The hole created by the pump could decay by an Auger process, and the decay was monitored in real time. Moreover, we saw quantum interferences in the signal, reflecting that the hole created by the probe pulse could be created in two different ways. The loss in coherence could also be followed in real time.

In this work, we did not include relaxation dynamics in the ion after the initial hole creation. The response to the removal of a krypton $3 p$ electron was studied in in Ref. [30], where it was shown that multielectron effects give rise to oscillations in the hole occupation on a 100 as time scale. The attosecond pump-attosecond probe technique is capable of resolving this time scale and should hence be able to resolve such multielectron relaxation processes, especially if the pump pulse is shorter than what is considered here. In this sense the present work outlines an experimental protocol to test the response of the atom on an attosecond time scale.

\section{ACKNOWLEDGMENTS}

This work was supported by NordForsk (Project No. 27861), the Danish Research Council (Grant No. 10-085430), the Swedish Research Council (VR), and the Göran Gustafsson Foundation. L.B.M. acknowledges useful discussions with S. R. Keiding, H. Stapelfeldt, and P. Balling, Aarhus University, Denmark.
[1] M. Uiberacker et al., Nature (London) 446, 627 (2007).

[2] A. L. Cavalieri et al., Nature (London) 449, 1029 (2007).

[3] L. Miaja-Avila, G. Saathoff, S. Mathias, J. Yin, C. La-o-vorakiat, M. Bauer, M. Aeschlimann, M. M. Murnane, and H. C. Kapteyn, Phys. Rev. Lett. 101, 046101 (2008).

[4] J. Mauritsson, P. Johnsson, E. Mansten, M. Swoboda, T. Ruchon, A. L'Huillier, and K. J. Schafer, Phys. Rev. Lett. 100, 073003 (2008).

[5] J. Mauritsson et al., Phys. Rev. Lett. 105, 053001 (2010).

[6] E. Goulielmakis et al., Nature (London) 466, 739 (2010).

[7] G. Porter and M. R. Topp, Nature (London) 220, 1228 (1968).

[8] M. Dantus, M. J. Rosker, and A. H. Zewail, J. Chem. Phys. 87, 2395 (1987).

[9] C. Rulliere, T. Amand, and X. Marie, in Femtosecond Laser Pulses-Principles and Applications, 2nd ed., edited by C. Rulliere, Chap. 8 (Springer Verlag, New York, 2005).

[10] P. Tzallas, E. Skantzakis, L. A. A. Nikolopoulos, G. D. Tsakiris, and D. Charalambidis, Nat. Phys. 7, 781 (2011).

[11] M. Drescher, M. Hentschel, R. K. M. Uiberacker, V. Yakovlev, A. Scrinzi, Th. Westerwalbesloh, U. Kleineberg, U. Heinzmann, and F. Krausz, Nature (London) 419, 803 (2002).

[12] C. Buth and R. Santra, Phys. Rev. A 75, 033412 (2007).

[13] S.-K. Son, L. Young, and R. Santra, Phys. Rev. A 83, 033402 (2011).

[14] J. C. Baggesen and L. B. Madsen, J. Phys. B 44, 115601 (2011).

[15] H. Wang, M. Chini, S. Chen, C.-H. Zhang, Y. Cheng, F. He, Y. Cheng, Y. Wu, U. Thumm, and Z. Chang, Phys. Rev. Lett. 105, 143002 (2010).
[16] R. W. Boyd, Nonlinear Optics, 3rd ed. (Elsevier, Amsterdam, 2008).

[17] R. Santra, V. S. Yakovlev, T. Pfeifer, and Z.-H. Loh, Phys. Rev. A 83, 033405 (2011).

[18] T. Mooney, E. Lindroth, P. Indelicato, E. G. Kessler, and R. D. Deslattes, Phys. Rev. A 45, 1531 (1992).

[19] P. Indelicato and E. Lindroth, Phys. Rev. A 46, 2426 (1992).

[20] P. Indelicato, S. Boucard, and E. Lindroth, Eur. Phys. J. D 3, 29 (1998).

[21] S. Salomonson and P. Öster, Phys. Rev. A 40, 5548 (1989).

[22] R. C. Cowan, The Theory of Atomic Structure and Spectra, Los Alamos Series in Basic and Applied Sciences No. 3 (University of California Press, Berkeley, 1981).

[23] G. Breit, Phys. Rev. 39, 616 (1932).

[24] E. Lindroth and A.-M. Mårtensson-Pendrill, Phys. Rev. A 39, 3794 (1989).

[25] I. Lindgren, J. Phys. B 23, 1085 (1990).

[26] R. D. Deslattes, E. G. Kessler, P. Indelicato, L. de Billy, E. Lindroth, and J. Anton, Rev. Mod. Phys. 75, 35 (2003).

[27] NIST database, http://www.nist.gov/pml/.

[28] E. Lindroth, Phys. Rev. A 49, 4473 (1994).

[29] R. van Leeuwen and E. J. Baerends, Phys. Rev. A 49, 2421 (1994).

[30] J. Breidbach and L. S. Cederbaum, Phys. Rev. Lett. 94, 033901 (2005).

[31] S. Pabst, L. Greenman, P. J. Ho, D. A. Mazziotti, and R. Santra, Phys. Rev. Lett. 106, 053003 (2011). 